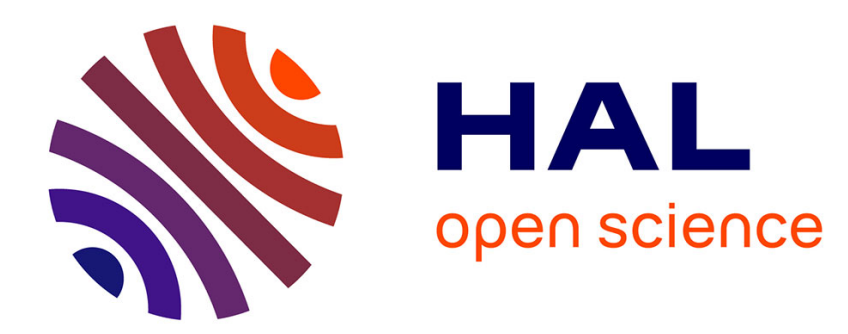

\title{
Les pays tropicaux dans les livres de géographie: manuels de l'enseignement secondaire entre 1925 et 1960 \\ Hervé Théry
}

\section{To cite this version:}

Hervé Théry. Les pays tropicaux dans les livres de géographie: manuels de l'enseignement secondaire entre 1925 et 1960. Espace Géographique, 1988, XVII (4), pp.299-306. halshs-00258036

\section{HAL Id: halshs-00258036 \\ https://shs.hal.science/halshs-00258036}

Submitted on 20 Feb 2008

HAL is a multi-disciplinary open access archive for the deposit and dissemination of scientific research documents, whether they are published or not. The documents may come from teaching and research institutions in France or abroad, or from public or private research centers.
L'archive ouverte pluridisciplinaire HAL, est destinée au dépôt et à la diffusion de documents scientifiques de niveau recherche, publiés ou non, émanant des établissements d'enseignement et de recherche français ou étrangers, des laboratoires publics ou privés. 


\section{Les pays tropicaux dans les livres de géographie : les manuels de l'enseignement secondaire entre 1925 et 1960}

Texte paru dans dans la revue L'Espace Géographique, pp. 299-306, tome XVII n 4, 1988. avec des figures en noir et blanc

\section{Résumé}

Les manuels de géographie témoignent de l'état de la science à une époque donnée, mais leur organisation est étroitement déterminée par les textes officiels et leur contenu très influencé par l'état de la société: entre 1925 et 1960 les pays tropicaux sont avant tout des colonies à exploiter.

\section{Mots-clés}

Manuels scolaires, histoire de la géographie, géographie coloniale.

Les manuels de géographie ne sont pas une sous-littérature. On n'en retiendra pas forcément pour preuve le fait de trouver sur leurs pages de garde, quand on examine ceux de ces années 1925 à 1960, quelques-uns des plus grand noms de la géographie de l'époque (Pierre Birot, Jean Brunhes, André Perpillou, André Cholley, Jean Dresch, Pierre George, etc.). Mais on peut avancer qu'ils sont un des meilleurs moyens de connaître la géographie telle qu'elle se fait réellement: bien souvent, ils sont l'unique "livre" qu'un citoyen français ait eu à sa disposition dans toute sa vie.

Aucune production géographique n'a été aussi lue que ces manuels, ils ont été mis entre les mains de tous les élèves, utilisés et commentés dans les cours - obligatoires - de géographie de l'enseignement primaire et secondaire. Et comme il s'agit d'y exprimer simplement des idées que l'on juge essentielles, le corps de doctrine central de la discipline, on y grossit souvent le trait, ce qui en fait parfois une caricature, mais permet sûrement de mieux repérer les traits saillants, les certitudes, les lacunes aussi. La "géographie" s'y exprime pleinement, plus que dans les cours universitaires, pas toujours publiés, et où le souci pédagogique est moins marqué.

Cette étude repose sur l'analyse d'un certain nombre de manuels destinés à l'enseignement secondaire, parce que leurs auteurs sont, plus souvent que dans l'enseignement élémentaire, des géographes reconnus (et non des pédagogues professionnels), parce que leur volume est plus étoffé. Il faut noter que ce choix introduit un biais, étant donné la minceur des effectifs de l'enseignement secondaire à l'époque: même si son recrutement social s'est élargi au cours de ces années, l'enseignement secondaire reste plus destiné à de futurs cadres qu'à la masse de la population: les Instructions de 1925 le disent nettement, qui voient dans les bacheliers à former "de", une liste où seule la présence des agriculteurs étonne et détonne.

L'analyse qui suit ${ }^{1}$ porte sur la vision que ces manuels donnent des pays tropicaux, au sens défini par Pierre Gourou. Ce cadre et cette définition ont été choisis parce que l'hypothèse de départ était que la façon dont les manuels de géographie présentent ces pays aurait dû être renouvelée par l'œuvre de ce géographe, publiée à peu près au milieu de la période concernée. Chemin faisant, il est apparu que fait le plus prégnant, celui qui pèse le plus sur les contenus et l'organisation des manuels, n'était toutefois pas celui-là, mais bien le programme officiel qui fixe, parfois de façon très précise, le contenu des manuels. Enfin il a été vite évident que la présentation de ces pays était déterminée par le fait que cet ensemble a été le champ principal de l'expansion coloniale: la période choisie voit l'apogée et la disparition de l'Empire colonial français 2 .

\section{Une certaine idée de la géographie}

Les auteurs des manuels, et avant eux les inspecteurs qui rédigent les Instructions officielles, ont dès le début de cette période le sentiment que 
la géographie a changé. Les Instructions officielles de 1925 l'affirment déjà:

"Peu de sciences ont sans doute fait plus de progrès à notre époque que la géographie. C'est seulement depuis environ un demi-siècle qu'elle a pris vraiment conscience de son objet et déterminé la méthode qui lui permettait de le réaliser".

L'idée maîtresse est que la géographie ne doit plus se contenter d'être une description. Celle-ci reste nécessaire, mais comme l'affirment ces mêmes Instructions:

"énumérer les faits géographiques, les décrire, ce n'est qu'une partie de ce que l'enseignement géographique comporte ... la description géographique appelle l'explication: elle l'amorce",

et plus loin elles précisent que:

"la description géographique n'est pas la description littéraire. Elle est courte, verveuse, précise, toute objective, réduite aux traits caractéristiques qui constituent vraiment l'individu géographique".

Cette idée d'une géographie " discipline " est reprise dans beaucoup des textes méthodologiques (Préfaces, avertissements, avant-propos, introductions, etc.), qui ouvrent les livres, dont on ne citera ici qu'un exemple, tiré d'un des plus anciens:

"Depuis la fin du XIXe siècle, tout l'effort de l'école géographique française a tendu à mettre en lumière la dépendance mutuelle des phénomènes géographiques... L'enseignement et les livres de Vidal de La Blache ont définitivement assuré le triomphe de ces idées. Ainsi comprise la géographie devient vraiment une science, c'est-à-dire un ensemble cohérent de phénomènes qui s'expliquent les uns par les autres" [Alcan, Première, 1931].

Vastes ambitions donc, qui n'apparaissent pourtant guère dans les textes qui constituent le corps des ouvrages. Même dans un des cas où l'on attendrait une vision synthétique, les cha- pitres de "généralités" qui introduisent à l'étude des continents, en classe de Cinquième, tous les manuels consultés, à deux ou trois exceptions près, ouvrent l'un après l'autre les sempiternels "tiroirs", et en reviennent à la géographie-inventaire que les préfaces dénoncent.

Du moins le contenu de ces tiroirs change-t-il ? On peut l'examiner sur un cas précis, la présentation générale du relief de l'Afrique, qui évolue évidemment entre 1925 et 1960. En 1925 et dans les années qui suivent, la première question abordée est encore celle des contours de l'Afrique, une tradition qui remonte à celle des vieux portulans, et l'on s'efforce de définir la forme du continent, l'accent étant mis sur les principaux accidents topographiques:

"L'Afrique est formée de plateaux que creusent des cuvettes et que des montagnes, souvent très hautes, séparent d'un littoral peu abordable" [Hachette, Cinquième, 1931].

Pourtant la connaissance du continent a progressé, comme en témoigne le manuel publié par Delagrave en 1934: l'Afrique est

"une très vieille terre, partiellement rajeunie par des effondrements, de failles et des volcans...un continent extrêmement ancien que les eaux marines n'auraient plus recouvert depuis les temps primaires".

En 1956 enfin le manuel publié aux éditions de l'école analyse l'extension du socle ancien réduit à l'état de pénéplaine, les formes sédimentaires, les mouvement tectoniques. On pourrait de la même façon souligner les progrès de la connaissance climatique ou pédologique, mais il continue à manquer une vision globale des milieux tropicaux et de leur originalité. Les travaux des géographes, qui prennent conscience dans les années 30 et 40 , de l'originalité des processus morphogénétiques et pédogénétiques sous climat tropical et équatorial, n'apparaissent guère dans les manuels, et moins encore une conception plus ample d'une géographie zonale 3 . Si le contenu des "tiroirs" s'améliore, à mesure que les connaissances analytiques progres- 
sent, les cadres anciens continuent à s'imposer dans les manuels, ce qui aggrave l'effet de fractionnement introduit par les programmes. Ce qui n'était pas un obstacle bien gênant en début de période, quand la géographie universitaire ellemême en était encore, pour ces pays, qu'au stade de l'inventaire, devient franchement nuisible quand se font jour des synthèses et des visions plus globales, qui ne trouvent pas leur place dans les manuels. La meilleure preuve en est que si Pierre Gourou peut exposer très clairement ses conceptions dans les chapitres qu'il consacre en 1961 à l'Afrique Française (cadre incommode mais obligé, puisqu'il s'agit d'un manuel de Première) il suit le plan classique, se permettant seulement, innovation notable, de traiter le climat avant le relief. Des programmes trop détaillés deviennent ici un réel obstacle à une présentation moderne de régions dont la connaissance a progressé, tandis que les textes officiels n'évoluaient pas.

\section{Le cadre contraignant des pro- grammes}

Les manuels scolaires mis sur le marché dépendent étroitement des programmes scolaires : pour être vendus, ils doivent en respecter scrupuleusement la lettre et l'esprit, sous peine d'être, sinon mis à l'index, du moins déconseillés par les inspecteurs, dont les moyens pression sur les professeurs, prescripteurs d'achat, sont multiples. Il suffit pour s'en convaincre, d'observer la coïncidence entre la parution de nouveaux programmes et le lancement de nouvelles générations de manuels (figure 1).

Les programmes qui régissent l'enseignement de la géographie, et donc le contenu des manuels, sont au nombre de 8 pour la période. Ils paraissent en $1925,1931,1937,1938,1941,1943,1944$ et 1945, un neuvième, paru en 1960, la terminant. Huit changement, ou plutôt sept, puisque celui de 1941 n'a pas eu d'effet sur la géographie: le régime de Vichy y réformait l'histoire et la philosophie, matières dangereuses, mais pas la géographie, sans doute jugée plus anodine. Tous les niveaux d'enseignement ne sont pas toujours touchés, et il s'agit parfois plus de retouches que de réformes, si bien qu'en fait quatre étapes majeures sont à retenir : 1925/31, 1937/38, 1944/45, 1960. La figure résume les changements les plus importants, qui ne concernent que le premier cycle puisque le dispositif du second cycle restant inchangé à quelques changements d'intitulés près: géographie générale en Seconde, la France en Première, les grandes puissances du Monde en Terminale.

\section{Figure 2: Les principales modifications des programmes}

1925-1931

1937-1938

1944-1945

1960

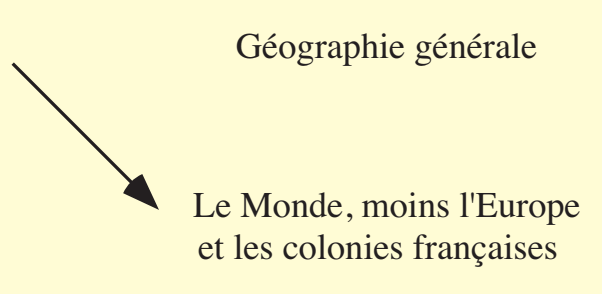

L'Europe

$4^{\text {e }} \quad$ La France et ses Colonies

$3^{\mathrm{e}}$

\begin{abstract}
Géographie générale + Amérique et Autralasie

Asie, Insulinde, Afrique
\end{abstract}

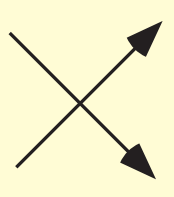

La France métropolitaine

et la France d'Outre-Mer

Géographie générale + Afrique

Pôles, Amérique, Asie, Océanie

L'Europe

La communauté 
Si l'on ajoute que les programmes de 1925 reprennent pour l'essentiel ceux de 1902, euxmêmes proches de ceux qui avaient été établis sous Jules Ferry, on ne peut manquer d'être frappé par la permanence du dispositif général ${ }^{4}$.

Quelle en est la logique ? Elle est au moins triple, scientifique (les diverses branches de la géographie, générale, humaine, régionale, économique), pédagogique (organisation en deux cycles complets, ce que les Instructions de 1938 soulignent avec force) et concentrique (pour le premier cycle du moins, du Monde à la France en passant par l'Europe, une logique établie par le changement de programme de 1938). Il est à noter que quand la nécessité de décharger la classe de Cinquième, alourdie par cette réforme, oblige à transférer l'étude d'une partie du Monde en Sixième, ce n'est plus "L'Amérique-Australasie" qui est détachée, mais l'Afrique, sans doute privilégiée parce qu'elle est le domaine majeur de la colonisation française.

Ces contraintes des programmes annuels sont aggravées par des Instructions souvent tatillonnes, qui prévoient dans le moindre détail la façon d'aborder chaque leçon (une heure de cours), fixent des plans-types, d'une minutie telle que les points qu'elles énumèrent représentent chacun à peu près cinq minutes de cours.

Face à ces contraintes, quelle est l'attitude des auteurs ? Les uns prennent le parti de suivre pas à pas le programme, d'autres affirment vouloir s'en détacher:

"Nous avons délibérément renoncé au procédé traditionnel qui emprisonne la connaissance des pays dans le moule uniforme et immuable: relief, climat et végétation, hydrographie, agriculture et industrie, voies de communication" [Baillière, Cinquième, 1935],

Mais ces prises de position d'ordre général ne concernent que rarement les régions tropicales. Une exception n'en est que plus remarquable:

"Nous avons pensé que cette redistribution des connaissances était indispensable à un moment où le concept continental devenait plus important, d'autant que le programme de 5e était le seul dont le libellé incitât à suivre cette voie" [Nathan,
Cinquième, 1952].

Ce manuel est d'ailleurs le seul, avec celui que dirigea André Cholley, en 1935 chez Baillière, à suivre un plan différent de celui que suggèrent les Instructions, bien que cette année soit celui où la marge de manœuvre est la plus grande: partout ailleurs le "plan" et la subdivision par pays achève la fragmentation introduite par les programmes annuels.

Passant sous les fourches caudines des programmes officiels, par respect naturel des textes émanant des autorités compétentes (bien naturelle puisqu'ils sont pour la plupart fonctionnaires du Ministère qui les édicte) et par la volonté de l'éditeur (soucieux de pouvoir écrire au frontispice de ses ouvrages "conforme"), les auteurs donnent donc de la zone tropicale une image en miettes, lui accordent une place des plus réduites.

Au total l'étude des pays qui la composent est très limitée: la classe de Cinquième, la fin de l'année de Sixième, quelques mentions dans les programmes de Géographie générale (Sixième et Seconde) et en Terminale, moins parmi les "grandes" (à l'exception du Brésil) que sous la rubrique "producteurs", pour quelques matière premières. Une place à part est toutefois faite aux colonies françaises, étudiées avec la métropole, en Troisième et en Première, secondairement aux colonies des autres pays européens: les pays indépendants disparaissent à peu près complètement, notamment l'Amérique latine.

La préface du manuel de Première paru chez Alcan en 1930 souligne les défauts de ce dispositif:

"Il arrive que des élèves connaissent assez solidement la France, mais soient très ignorants de la géographie, surtout de la géographie physique, des autres régions du Monde"

L'auteur se rassurait en partie en poursuivant:

"La variété de notre domaine colonial permet au professeur de remédier, dans une certaine mesure, à cet inconvénient"

C'est là une idée que l'on retrouve sous la plume de L. François et R. Mangin , qui appellent à: 


\section{Figure 1: Les éditeurs sur le marché}

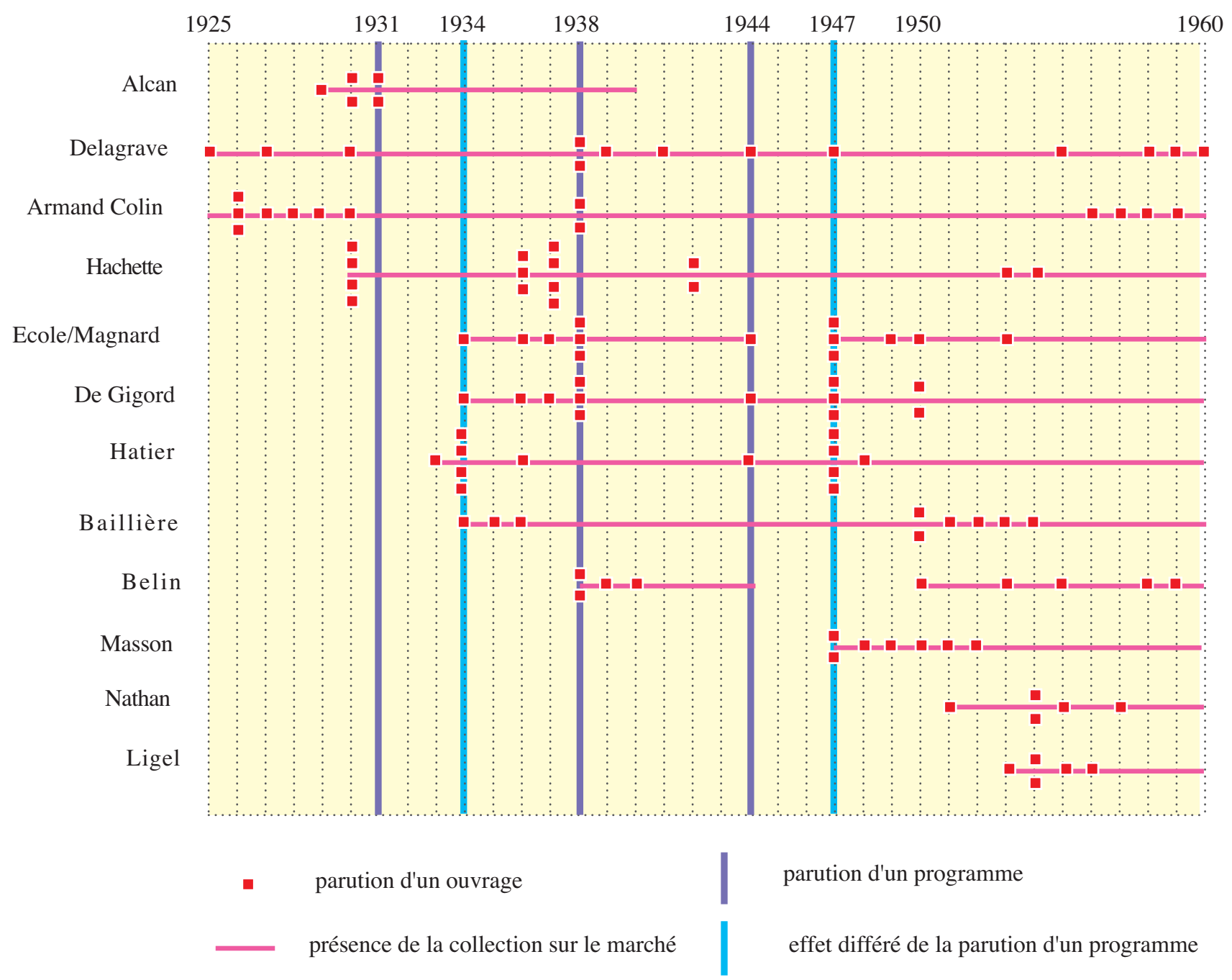

Les manuels paraissent en général par familles, couvrant les deux cycles, de la Sixième à la Terminale, ou au moins par cycles entiers. La date de leur lancement dépend étroitement de la parution de nouveaux programmes, il la suit immédiatement ou parfois avec un décalage, comme en 1934 ou en 1947: serait-ce pour cause de crise économique, ou de rationnement du papier ?

S'il existe des familles de manuels, les "cours" baptisés du nom de leur directeur, Varon (Armand Colin), Jean Brunhes (Hatier), Gallouedec et Maurette ou Demangeon (Hachette), il existe aussi des générations d'éditeurs, comme le montre ce tableau. Tous ne sont pas présents, avec des collections actives, en même temps (on a supposé ici qu'une collection se poursuivait tant qu'elle était connaissait des rééditions). Si certains sont présents d'un bout à l'autre de la période (Delagrave, Armand Colin), d'autres disparaissent (Alcan, apparaissent ou réapparaissent après des éclipses. La concurrence, toujours forte, s'accroit donc au fil du temps, à mesure que le marché s'élargit.

“insister sur les territoires d'Outre-mer, dans lesquels nous trouvons toute la diversité du Monde". [Hachette, Première, 1943].

Cet appel à l'Empire pour corriger les défauts liés à l'architecture générale des programmes dit assez que le fait colonial l'emporte dans ces manuels sur les considérations scientifiques. L'analyse des contenus le confirme nettement. 


\section{Un point de vue extérieur}

Il est d'abord présent dans la façon même dont les auteurs présentent les pays qu'ils ont à faire connaître. Le biais le plus curieux est que ces pays sont encore, semble-t-il, vu du bateau qui amène les navigateurs européens, ou à la rigueur du point de vue des explorateurs qui ont à les parcourir. D'où le souci de décrire, les côtes, les ports et abris que l'on peut y trouver, les fleuves qui facilitent la pénétration (et non l'accès au littoral ou l'exportation):

"Les fleuves d'Afrique ont des embouchures médiocrement favorables... [ils] n'offrent que des voies insuffisantes à la navigation" [Hachette, Cinquième, 1931] "coupés de rapides et ayant un régime généralement très inégal, les fleuves africains sont de médiocres voies de pénétration" [Alcan, Cinquième, 1931].

Au moins jusqu'en 1935, les pays tropicaux sont donc vus de l'extérieur, alors que les pays tempérés le sont de l'intérieur (le chapitres sur les côtes y est alors le dernier, alors qu'il est souvent le premier pour les pays tropicaux). Ensuite, à mesure que l'aviation commerciale se développe, on voit le monde tropical comme on y débarque: de haut.

Il reste pourtant quelque chose de cette vision depuis l'extérieur dans l'insistance mise à voir dans l'Afrique " un monde fermé ", "le plus fermé de tous les continents", aux climats "peu hospitaliers", voire "répulsifs": ce dernier terme, qui s'applique bien à quelqu'un qui vient de l'extérieur (et est mal accueilli), témoigne de ce que les point de vue est bien celui d'un colon, réel ou potentiel, plus que celui des autochtones, et il est remarquable que ces plaintes concernent presque toujours l'Afrique, où s'est porté le gros de l'effort colonial français, rarement l'Asie, et jamais l'Amérique.

Une citation résume bien ce point de vue sur l'Afrique, celui d'un continent fermé, à conquérir, le résumé donné en fin de chapitre, la quintessence de ce qu'il faut en retenir:

“I- L’Afrique, monde fermé.
L'Afrique, continent massif, aux rivages rectilignes, est peu accueillante. Elle présente sur tout son pourtour de hautes barrières montagneuses (chaînes plissées au Nord et au Sud, rebords de plateaux dans le reste du continent). Ses côtes, généralement plates, sont exposées de plein fouet aux tempêtes et difficile d'abord (barre), ses climats (tropicaux et équatoriaux) sont malsains, seul le Nord et le Sud (climats subtropical) sont habitables par les Blancs.

Les Musulmans sont réfractaires à la civilisation européenne, les Noirs ne représentent que de médiocres auxiliaires pour la mise en valeur du pays.

II- L'Afrique, terre de colonisation.

L'Afrique tout entière (sauf l'Egypte, le Libéria et l'Ethiopie) est partagée entre les puissances européennes. Elle a attiré la colonisation aux XIXe et XXe siècles parce que c'est un pays aux ressources variées, où les indigènes incapables de concurrencer les européens constituent au contraire pour eux une clientèle assurée" [Baillière, Cinquième, 1935].

Car une fois installé, reste à inventorier les ressources disponibles. C'est généralement l'étape suivante, et les manuels manquent rarement de le faire, comme le faisaient sur le terrain les envoyés des Sociétés de Géographie, avantgarde de la colonisation. La géographie est ici une science utile, appliquée. L'inventaire est centré sur les produits exploitables, vise avant tout à permettre de classer la colonie dans les cadres bien établis:

"Les pays de climat sain sont des colonies de peuplement ... les colonies de pénétration permettent l'accès vers les pays riches et peuplés, susceptibles d'entrer en relations commerciales actives avec la métropole. Les colonies d'exploitation sont celles où, par la suite du caractère malsain du climat, l'Européen n'a pu s'établir 
d'une façon permanente, mais où les richesses $\mathrm{du}$ sol et du sous-sol fournissent un aliment abondant au commerce d'exportation [Alcan, Cinquième, 1926].

Les manuels reprennent ici le point de vue des administrateurs coloniaux, et vont parfois jusqu'à porter des jugements de valeur sur les puissances rivales:

"L'Angleterre s'est là encore taillé la part du lion, en nous évinçant d'Egypte, en profitant des guerres de la Révolution pour mettre la main sur le Cap, en brisant l'indépendance des républiques boers" [Hatier, Cinquième, 1934].

Le jugement est plus dur encore quand le rival est aussi le nouvel ennemi héréditaire,, vaincu de surcroît:

“ Edifiées sur les méthodes de l'administration coloniale allemandes, sur les répressions les plus cruelles, les réquisitions arbitraires et toutes le formes de travail forcé qui ont dépeuplé de vastes étendues de territoire dans l'est africain et le Cameroun, sans parler du sort tragique et bien connu des Hereros du Sud-Ouest Africain Allemand, les Puissances Alliées n'ont pas hésité à déclarer «la faillite de l'Allemagne dans le domaine de la civilisation coloniale» et à proclamer sa déchéance pour le plus grand bien des indigènes" [Delagrave, Cinquième,1934].

Parfois aussi ils tentent de se consoler de n'avoir pas eu la meilleure part, de n'avoir pas par exemple plus de colonies de peuplement:

"Nous en avons peu: aussi bien seraient-telles inutiles à un pays comme la France, où la population s'accroît lentement" [Alcan, Cinquième, 1926].

\section{Des colonies à exploiter}

La France ayant donc conquis cet Empire, formé surtout de "colonies d'exploitation", reste à les exploiter: c'est ce point de vue que les auteurs adoptent très naturellement et veulent faire partager aux élèves qui les liront. Parmi les ressources à inventorier figure donc en bonne place la capacité de travail de la main d'œuvre indigène, indispensable pour mettre en valeur les autres ressources. Le point de vue est ici clairement celui de l'administrateur ou de l'entrepreneur, exposé en toute franchise - ou en toute naïveté? - aux élèves:

"Dans les riches régions littorales, la population indigène est trop clairsemée et trop paresseuse pour fournir la main d'œuvre nécessaire à une grande exploitation commerciale"

[Hatier, Cinquième, 1934].

"Ils se plient difficilement aux disciplines qu'impose une civilisation plus perfectionnée: c'est pourquoi on leur reproche parfois d'être paresseux" [Baillière, Cinquième, 1935].

La situation est ici encore assez particulière en Afrique, et moins favorable que dans d'autres colonies françaises, comme l'Indochine, où:

"La main d'œuvre indigène se trouve en abondance et surtout à très bon marché, le «facteur humain» se trouve en Indochine à des conditions beaucoup plus favorables à l'exploitation européenne qu'en Afrique occidentale" [Alcan, Cinquième, 1926].

En Afrique en effet, il faute employer des subterfuges:

"Il est possible de les attirer dans les plantations ou dans les mines, où ils doivent effectuer un dur labeur. Le Noir aime la parure, l'objet inconnu qui lui paraît un luxe. En lui offrant un salaire, si modique soit-il, qui lui permette de satisfaire son désir, on l'attire vers les travaux les plus durs" [Baillière, Cinquième, 1935].

Mais la vraie solution est à plus long terme, et passe par des entreprises apparemment sans intérêt économique:

"Les nègres africains... sont à la fois trop peu nombreux, trop peu travailleurs et trop peu instruits pour fournir actuellement les instruments d'une exploitation intensive. Repeupler 
l'Afrique par la diminution de la mortalité infantile, donner aux nègres le goût du travail, leur inculquer des connaissances techniques élémentaires, c'est une œuvre de longue haleine que la multiplication des dispensaires et des écoles mettra en bonne voie." [Alcan, Cinquième, 1926].

Le lien est donc établi entre la mission civilisatrice et les bénéfices à à tirer de la colonies, comme cela est parfois candidement noté:

"Il est du devoir de tous les Français de contribuer dans la mesure de leurs forces à la propagation de leur langue, par laquelle se répandent par le monde les idées généreuses qui font aimer la France et qui accroissent son autorité morale. En outre la diffusion de notre langue aide puissamment la diffusion de nos produits" [Alcan, Cinquième, 1926].

Cela donne une tonalité nouvelle aux déclarations classiques -et très probablement sincères - sur les motifs réels de la colonisation française:

"La France, grâce à la hardiesse de ses explorateurs et de ses officiers, s'est taillé un vaste Empire, nécessaire à son commerce, plus nécessaire à sa défense, mais où les préoccupations de civilisation ... passent avant celles de l'exploitation" [Hatier, Cinquième,, 1934].

Les justifications avancées sont parfois étranges, comme celles qui expliquent l'intervention européenne par la nécessité de mettre fin à l'esclavage:

“Des marchands d'esclaves organisaient des battues humaines et ramenaient de longues files de captifs, destinés soit aux monarchies despotiques de l'Islam, soit aux plantations de coton américaines. Ce sera l'honneur de l'Europe d'avoir entravé et arrêté ce commerce odieux, répugnant, de l'homme par l'homme" [Delagrave, Cinquième, 1934].

Voilà un paragraphe qui a du faire chaud au cœur des petits Bordelais ou des petits Nantais dont les ancêtres s'étaient enrichis dans le com- merce du bois d'ébène.

Il arrive que l'on aille plus loin dans les jugements de valeur, tombant dans un racisme sans fard:

“Dans les régions du bananier, le Nègre n'a qu'à se laisser vivre, sans se donner d'autre peine que de cueillir les fruits des arbres. Comme il n'est pas poussé par la nécessité, il est mou, indolent, corrompu et lâche, et si le besoin de viande se fait trop impérieusement sentir, il se nourrit de chair humaine: il est anthropophage"

Ces habitants des régions glacées ont l'air engourdi dans leurs vêtement de peaux de bêtes: ils sont tristes, souffreteux, et d'intelligence peu développée"

"Les Japonais, à qui on ferait gravement injure en les prenant pour des Chinois, sont généralement bien proportionnés, le crâne volumineux. On distingue parmi eux deux types, un type fin, de taille élancée et de nez droit, dans les hautes classes de la société, et un type grossier, le corps trapu, la face large, les pommettes saillantes et le nez aplati, dans la masse du peuple"

[Delagrave, Cinquième, 1934].

La date de ces citations correspond à une apogée du système colonial, juste après la grande Exposition Coloniale de 1931 qui avait rassemblé à Paris des échantillons, architecturaux et humains, de tout l'Empire. On n'en trouve plus d'aussi caricaturales après cette date, à mesure que la guerre et la décolonisation ébranlent l'Empire, les auteurs se détachent du point de vue colonial. Il ne remettent pourtant pas en question l'œuvre accomplie, comme en témoignent deux citations de la fin de la période:

"La France transforme les pays d'outre-mer...

a) elle améliore les conditions de vie, par la lutte contre la maladie et l'ignorance.

b) elle transforme leur économie.

c) la France assure des débouchés aux produits 
tropicaux.

d) La France fournit capitaux et techniciens.

[Hatier, Cinquième, 1958].

"Au second empire colonial du monde, après le Commonwealth, [la Communauté] a apporté, malgré bien des difficultés, les bienfaits d'une civilisation généreuse. Comme les autres nations civilisatrices, elle a longtemps vu dans ces territoires des comptoirs qui devaient fournir des matières premières et des débouchés à l'industrie métropolitaine. Mais en même temps, elle a rempli sa mission éducatrice des peuples d'Outre-mer. Elle les a initiés à la civilisation européenne, c'est-à-dire au progrès matériel et humain" [Ecole, Cinquième, 1959].

\section{Géographie tropicale, géographie coloniale, géographie datée}

Partis à la recherche de la tropicalité, nous avons donc surtout trouvé dans les manuels une géographie coloniale, influencée par des programmes eux-mêmes marqués par l'histoire coloniale: par la place privilégiée faite aux colonies françaises, par l'accent mis sur les ressources exportables, y compris le travail des indigènes, par la défense et illustration de l'œuvre coloniale, ces manuels, à de rares exceptions près, ont contribué à justifier l'expansion française outre-mer. En cela ils ne se distinguent pas d'autres manuels, ni d'ailleurs de l'opinion majoritaire dans le pays. Si les manuels scolaires sont certainement dans la géographie, ils sont aussi dans leur temps, dans son idéologie.

Ils sont aussi, étant donné le poids des programmes, des Instructions Officielles, et donc de l'Inspection Générale, partie intégrante d'une institution scolaire très dépendante de l'Etat, très jacobine et pointilleuse. A cela les auteurs peuvent difficilement échapper, aujourd'hui comme hier. Tout au plus peuvent-ils faire leur les idées que Jean Brunhes avançait en 1934, dans la Préface de la série de manuels qu'il dirigea chez Hatier:

"A nous il ne nous appartient de réformer ni les programmes, ni les examens. La solution à chercher ? Composer des manuels qui fassent pénétrer l'essentiel par des procédés plus expéditifs, plus démonstratifs, plus révélateurs".

\section{Notes}

${ }^{1}$ Ce texte est pour l'essentiel tiré d'un mémoire de mâ̂trise, rédigé en 1973 sous la direction de Philippe Pinchemel, à l'Université Paris I. Le fonds d'archive exploité était celui des manuels scolaires conservés par l'INRP. Un recensement aussi complet que possible des manuels parus à cette époque figure dans les annexes de ce mémoire, ainsi qu'une petit étude quantitative sur les manuels de la librairie Armand Colin.

2 Un autre mémoire, jamais terminé, devait couvrir la période 1870-1925.

3 Bien que l'article fondateur d'Emmanuel de Martonne, "Géographie zonale, la zone tropicale", soit paru dans les Annales de Géographie en 1946 ( $\mathrm{n}^{\circ}$ 297) et Les pays tropicaux de Pierre Gourou aient été publiés en 1947 aux Presses Universitaires de France.

4 qui n'a pas été tellement modifié par la suite, si ce n'est sous René Haby, et rétablie en grand partie par les programmes actuellement en vigueur. 